\title{
SURFACES IN $\mathbb{R}^{4}$ WITH CONSTANT AFFINE GAUSS MAPS
}

\author{
LUC VRANCKEN AND CHANGPING WANG
}

(Communicated by Christopher Croke)

\begin{abstract}
In this paper we study the affine Gauss maps of nondegenerate surfaces in $\mathbb{R}^{4}$ with respect to the Burstin-Mayer normalization (1927) and with respect to the normalization obtained by Nomizu and the first author in 1993. We determine up to affine transformations all surfaces in $\mathbb{R}^{4}$ with constant affine Gauss maps.
\end{abstract}

\section{INTRODUCTION}

The purpose of this paper is to study the Gauss maps of nondegenerate surfaces in $\mathbb{R}^{4}$ with respect to the Burstin-Mayer normalization [1] and with respect to the normalization obtained by Nomizu and the first author in [6]. The first normalization has the advantage of being closely related to the induced affine metric, while the second always induces an equiaffine structure on the surface. Another property of this second normalization with respect to extremal surfaces can be found in [2]. For a systematic study of the different normalizations we refer to [6] and [7].

Let $x: M \rightarrow \mathbb{R}^{4}$ be an immersed surface. For any local basis $\sigma=\left\{E_{1}, E_{2}\right\}$ for $M$ one can locally define a symmetric 2 -form

$$
\begin{aligned}
g^{\sigma}= & {\left[E_{1}(x), E_{2}(x), d E_{1}(x), d E_{2}(x)\right] } \\
& -\left[E_{1}(x), E_{2}(x), d E_{2}(x), d E_{1}(x)\right],
\end{aligned}
$$

where $[\ldots]$ is the standard determinant in $\mathbb{R}^{4}$. The conformal class of $g^{\sigma}$ is evidently independent of the choice of $\sigma . x$ is called a nondegenerate surface in $\mathbb{R}^{4}$ if $g^{\sigma}$ is nondegenerate. If we write $g^{\sigma}=\sum_{i, j} g_{i j}^{\sigma} \theta_{i} \otimes \theta_{j}$, where $\left\{\theta_{1}, \theta_{2}\right\}$ is the dual basis for $\left\{E_{1}, E_{2}\right\}$, then the so-called affine metric $g$ for nondegenerate surface $x$ is defined by

$$
g=\left(\operatorname{det}\left(g_{i j}^{\sigma}\right)\right)^{-1 / 3} g^{\sigma} .
$$

It is easy to see that $g$ is independent of the choice of $\sigma$ and therefore globally defined. By (1.1) and (1.2) we know that $g$ is an equiaffine invariant for $x$.

Received by the editors June 10, 1993.

1991 Mathematics Subject Classification. Primary 53A15.

Key words and phrases. Equiaffine surfaces in $\mathbb{R}^{4}$, affine Gauss maps.

The first author is a Senior Research Assistant of the National Fund for Scientific Research.

The second author was partially supported by the DFG-project "Affine Differential Geometry" at the TU Berlin. 
A nondegenerate surface is said to be definite (resp. indefinite) if $g$ is definite (resp. indefinite).

Let $x: M \rightarrow \mathbb{R}^{4}$ be a definite surface in $\mathbb{R}^{4}$. By exchanging the last two coordinates of $\mathbb{R}^{4}$ if necessary we may assume that the affine metric $g$ for $x$ is positive definite. We introduce a local complex coordinate $z$ for $(M, g)$ defined on an open set $U \subset M$; then $g=2 e^{\omega}|d z|^{2}$ for some function $\omega \in$ $C^{\infty}(U)$. We define

$$
\xi:=x_{z z}-\omega_{z} x_{z}: U \rightarrow \mathbb{C}^{4} .
$$

Since $x$ is nondegenerate, we can show that $\xi$ has no zero point. Furthermore, $\xi$ changes conformally if we take another complex coordinate. So we get a globally defined map $[\xi]: M \rightarrow \mathbb{C} P^{3}$. We call it the affine Gauss map for the definite surface $x$, which is clearly an equiaffine invariant.

For an indefinite surface $x$ we can introduce a local asymptotic coordinate system $(u, v)$ for $g$ defined on an open set $U \subset M$ with $g=2 e^{\omega} d u d v$. We define

$$
\xi=x_{u u}-\omega_{u} x_{u}, \quad \eta=x_{v v}-\omega_{v} x_{v} .
$$

Since $x$ is nondegenerate, we can show that both $\xi$ and $\eta$ have no zero point. Since $\xi$ and $\eta$ change conformally if we use another asymptotic coordinate system, we get two globally defined maps $[\xi],[\eta]: M \rightarrow \mathbb{R} P^{3}$. We call them the affine Gauss maps for the indefinite surface $x$ with respect to the BurstinMayer normalization, which are equiaffine invariants of $x$. Of course, for a different normalization we can define the corresponding affine Gauss maps $[\tilde{\xi}]$, $[\tilde{\eta}]: M \rightarrow \mathbb{R} P^{3}$ by taking the component of $\xi$ (resp. $\eta$ ) which belongs to the normal plane.

Our main results are the following:

Theorem 1. Let $x: M \rightarrow \mathbb{R}^{4}$ be a definite surface with constant affine Gauss map $[\xi]$. Then, up to affine transformations in $\mathbb{R}^{4}, x(M)$ is an open part of the surface in $\mathbb{R}^{4}$ given by

$$
\left\{\left(u, v, u^{2}-v^{2}, 2 u v\right) \mid(u, v) \in \mathbb{R}^{2}\right\} .
$$

Theorem 2. Let $x: M \rightarrow \mathbb{R}^{4}$ be an indefinite surface with constant affine Gauss maps $[\xi]$ and $[\eta]$ with respect to the Burstin-Mayer normalization. Then, up to affine transformations in $\mathbb{R}^{4}, x(M)$ is an open part of either

$$
\left\{\left(u, v, u^{2}, v^{2}\right) \mid(u, v) \in \mathbb{R}^{2}\right\}
$$

or

$$
\left\{\left(v, e^{-2 v}, e^{-2 v} u, e^{-2 v} u^{2}\right) \mid(u, v) \in \mathbb{R}^{2}\right\} .
$$

Theorem 3. Let $x: M \rightarrow \mathbb{R}^{4}$ be an indefinite surface with constant affine Gauss maps with respect to the Nomizu-Vrancken normalization. Then, up to affine transformations in $\mathbb{R}^{4}, x(M)$ is an open part of either

$$
\left\{\left(u, v, u^{2}, v^{2}\right) \mid(u, v) \in \mathbb{R}^{2}\right\}
$$

or

$$
\left\{\left(v,-\frac{4}{9} v^{2}+u^{4 / 3}, u^{2}, \frac{3}{4} v u^{4 / 3}-\frac{1}{9} v^{3}\right) \mid(u, v) \in \mathbb{R}^{2}\right\}
$$


or

$$
\left\{\left(u^{2} v^{2 / 3}, u v^{2 / 3}, v^{2 / 3}, v^{2}\right) \mid(u, v) \in \mathbb{R}^{2}\right\}
$$

We will prove Theorem 1 in $\S 2$, Theorem 2 in $\S 3$, and Theorem 3 in $\S 4$.

\section{THE PROOF OF THEOREM 1}

Let $x: M \rightarrow \mathbb{R}^{4}$ be a definite surface with positive definite affine metric $g$. We may assume that $M$ is simply connected, otherwise we consider the universal covering $\pi: M^{\prime} \rightarrow M$ and the immersion $x^{\prime}:=x \circ \pi: M^{\prime} \rightarrow \mathbb{R}^{4}$ with $x^{\prime}\left(M^{\prime}\right)=x(M)$.

Let $z=u+i v$ be a local complex coordinate for $(M, g)$ defined on a simply connected open set $U \subset M$. Then we have

$$
g=e^{\omega}(d z \otimes d \bar{z}+d \bar{z} \otimes d z):=2 e^{\omega}|d z|^{2} .
$$

By (1.1) with $\sigma=\left\{\partial_{u}, \partial_{v}\right\}$ and $\partial_{z}:=\frac{1}{2}\left(\partial_{u}-i \partial_{v}\right)$ we get

$$
\begin{aligned}
g^{\sigma} & =\left[x_{u}, x_{v}, d x_{u}, d x_{v}\right]-\left[x_{u}, x_{v}, d x_{v}, d x_{u}\right] \\
& =4\left(\left[x_{z}, x_{\bar{z}}, d x_{z}, d x_{\bar{z}}\right]-\left[x_{z}, x_{\bar{z}}, d x_{\bar{z}}, d x_{z}\right]\right) .
\end{aligned}
$$

Using the formula $d f=f_{z} d z+f_{\bar{z}} d \bar{z}$ we get

$$
\begin{aligned}
g^{\sigma}= & 8\left[x_{z}, x_{\bar{z}}, x_{z \bar{z}}, x_{z z}\right] d z \otimes d z+8\left[x_{z}, x_{\bar{z}}, x_{\overline{z z}}, x_{z \bar{z}}\right] d \bar{z} \otimes d \bar{z} \\
& -4\left[x_{z}, x_{\bar{z}}, x_{z z}, x_{\overline{z z}}\right](d z \otimes d \bar{z}+d \bar{z} \otimes d z) .
\end{aligned}
$$

Since $g^{\sigma}$ is conformal to $g$, by (2.1) and (2.2) we have

$$
\left[x_{z}, x_{\bar{z}}, x_{z \bar{z}}, x_{z z}\right]=\left[x_{z}, x_{\bar{z}}, x_{\overline{z z}}, x_{z \bar{z}}\right]=0, \quad\left[x_{z}, x_{\bar{z}}, x_{z z}, x_{\overline{z z}}\right] \neq 0 .
$$

Thus $x_{z \bar{z}}$ is tangential and $\left\{x_{z}, x_{\bar{z}}, x_{z z}, x_{\overline{z z}}\right\}$ is a local frame in $\mathbb{R}^{4} \otimes \mathbb{C}$ along $U$. By (2.1), (2.2), and (1.2) we get

$$
\begin{gathered}
g=-\left(\left[x_{z}, x_{\bar{z}}, x_{z z}, x_{\bar{z}}\right]\right)^{1 / 3}(d z \otimes d \bar{z}+d \bar{z} \otimes d z), \\
e^{3 \omega}=-\left[x_{z}, x_{\bar{z}}, x_{z z}, x_{\overline{z z}}\right] .
\end{gathered}
$$

Let $\tau$ be another complex coordinate for $(M, g)$ with

$$
g=e^{\rho}(d \tau \otimes d \bar{\tau}+d \bar{\tau} \otimes d \tau) .
$$

Then we have

$$
x_{z z}-\omega_{z} x_{z}=\left(x_{\tau \tau}-\rho_{\tau} x_{\tau}\right)\left(\frac{d \tau}{d z}\right)^{2} \neq 0 .
$$

Thus $[\xi]: M \rightarrow \mathbb{C} P^{3}$ defined by $\left.[\xi]\right|_{U}=\left[x_{z z}-\omega_{z} x_{z}\right]: U \rightarrow \mathbb{C} P^{3}$ is well defined. We call it the affine Gauss map of $x$.

Now we assume that the Gauss map $[\xi]$ is constant. Then we can find $\varphi \in C^{\infty}(U)$ such that

$$
\xi=x_{z z}-\omega_{z} x_{z}=e^{\varphi} v_{0}, \quad v_{0}=\text { const } \in \mathbb{C}^{4} .
$$

Since $\left\{x_{z}, x_{\bar{z}}, \xi, \bar{\xi}\right\}$ is a local frame in $\mathbb{C}^{4}$ along $U \subset M$ and $x_{z \bar{z}}$ is tangential, we can write the structure equations of $x$ as follows:

$$
\begin{array}{ll}
x_{z z}=\omega_{z} x_{z}+\xi, & x_{z \bar{z}}=\lambda x_{z}+\bar{\lambda} x_{\bar{z}} ; \\
x_{\bar{z} z}=\lambda x_{z}+\bar{\lambda} x_{\bar{z}}, & x_{\overline{z z}}=\omega_{\bar{z}} x_{\bar{z}}+\bar{\xi} ; \\
\xi_{z}=\varphi_{z} \xi, & \xi_{\bar{z}}=\varphi_{\bar{z}} \xi ; \\
\bar{\xi}_{z}=\bar{\varphi}_{z} \bar{\xi}, & \bar{\xi}_{\bar{z}}=\bar{\varphi}_{\bar{z}} \bar{\xi} .
\end{array}
$$


By (2.7) and the identity $x_{z z \bar{z}}=x_{z \bar{z} z}$ we get

$$
\omega_{z \bar{z}}=\lambda_{z}+|\lambda|^{2}, \quad \omega_{z} \bar{\lambda}=\bar{\lambda}_{z}+\bar{\lambda}^{2}, \quad \lambda=\varphi_{\bar{z}} .
$$

Thus we know that $\varphi_{z \bar{z}}=\omega_{z \bar{z}}-\left|\varphi_{\bar{z}}\right|^{2}$ is real, i.e., $(\operatorname{Im} \varphi)_{z \bar{z}}=0$. So we can find a holomorphic function $f(z)$ on $U$ such that $\operatorname{Im} \varphi=f(z)+\overline{f(z)}$.

Let $\tau$ be another complex coordinate for $(M, g)$ with

$$
g=e^{\rho}(d \tau \otimes d \bar{\tau}+d \bar{\tau} \otimes d \tau) .
$$

We denote $\eta=x_{\tau \tau}-\rho_{\tau} x_{\tau}$. Since the Gauss map is constant, we can find $\psi$ such that $\eta=e^{\psi} v_{0}$. By (2.5) and (2.6) we have

$$
\varphi=\psi+2 \ln \left(\frac{d \tau}{d z}\right) .
$$

Then $\operatorname{Im} \varphi=\operatorname{Im} \psi-i \ln \left(\frac{d \tau}{d z}\right)-\overline{i \ln \left(\frac{d \tau}{d z}\right)}$. Since $\operatorname{Im} \varphi=f(z)+\overline{f(z)}$, by letting $\tau=\int_{z_{0}}^{z} e^{i f(z)} d z$ on $U$ we get $\operatorname{Im} \psi=0$. Such a complex coordinate $\tau$ is uniquely determined up to a real positive factor. Thus by changing complex coordinate if necessary we may assume that $\operatorname{Im} \varphi=0$, i.e., $\varphi$ is a real function on $U$.

By (2.4) and (2.6) we have $\left[x_{z}, x_{\bar{z}}, \xi, \bar{\xi}\right]=-e^{3 \omega}$, which implies that $\bar{\lambda}+\varphi_{z}+$ $\bar{\varphi}_{z}=2 \omega_{z}$. Since $\varphi$ is real, from the last equation of $(2.11)$ we get $3 \varphi_{z}=2 \omega_{z}$. By (2.11) we obtain

$$
\omega_{z \bar{z}}=\frac{3}{4} \omega_{z} \omega_{\bar{z}}, \quad \omega_{z z}=\frac{1}{3} \omega_{z}^{2} .
$$

It follows from the identity $\omega_{z \bar{z} z}=\omega_{z z \bar{z}}$ that $\left|\omega_{z}\right|^{2}=0$, i.e., $\omega$ and $\varphi$ are constant. In particular, $g$ is flat. Since $M$ is simply connected, $M$ has to be noncompact. So we can take $U=M$. From (2.7) and (2.8) we obtain

$$
x_{z z}=\xi, \quad x_{z \bar{z}}=0 .
$$

The second equation of (2.14) implies that $x=h(z)+\overline{h(z)}$ for some holomorphic mapping $h: M \rightarrow \mathbb{C}^{4}$. Since $\xi$ is constant, the first equation of (2.14) gives $h(z)=z^{2} \xi+z \eta+c$ for some constant vectors $\eta, c \in \mathbb{C}^{4}$. Since $x$ is nondegenerate, $\{\xi, \bar{\xi}, \eta, \bar{\eta}\}$ are linearly independent. We denote by $A$ the matrix $A=(\eta+\bar{\eta}, i(\eta-\bar{\eta}), \xi+\bar{\xi}, i(\xi-\bar{\xi}))$ and $z=u+i v \in M$; then we have

$$
x=A^{t}\left(u, v, u^{2}-v^{2}, 2 u v\right)+(c+\bar{c}),
$$

i.e., $x$ is affinely equivalent to the surface $\left\{\left(u, v, u^{2}-v^{2}, 2 u v\right) \mid(u, v) \in \mathbb{R}^{2}\right\}$ in $\mathbb{R}^{4}$. Thus we complete the prove of Theorem 1 .

\section{THE PROOF OF THEOREM 2}

Let $x: M \rightarrow \mathbb{R}^{4}$ be an immersed surface with indefinite affine metric $g$. We may assume that $M$ is simply connected. Thus we can introduce a global asymptotic coordinate system $(u, v)$ for $g$ such that

$$
g=e^{\omega}(d u \otimes d v+d v \otimes d u), \quad \omega \in C^{\infty}(M) .
$$

From (1.1) with $\sigma=\left\{\partial_{u}, \partial_{v}\right\}$ we get

$$
\begin{aligned}
g^{\sigma}= & {\left[x_{u}, x_{v}, d x_{u}, d x_{v}\right]-\left[x_{u}, x_{v}, d x_{v}, d x_{u}\right] } \\
= & 2\left[x_{u}, x_{v}, x_{u u}, x_{u v}\right] d u \otimes d u+2\left[x_{u}, x_{v}, x_{u v}, x_{v v}\right] d v \otimes d v \\
& +\left[x_{u}, x_{v}, x_{u u}, x_{v v}\right](d u \otimes d v+d v \otimes d u) .
\end{aligned}
$$


Since $g^{\sigma}$ is conformal to $g$, we have

$$
\begin{aligned}
& {\left[x_{u}, x_{v}, x_{u u}, x_{u v}\right]=\left[x_{u}, x_{v}, x_{u v}, x_{v v}\right]=0,} \\
& {\left[x_{u}, x_{v}, x_{u u}, x_{v v}\right] \neq 0 .}
\end{aligned}
$$

Thus $x_{u v}$ is tangential. By (3.1)-(3.3), (1.2), and (1.4) we get

$$
\left[x_{u}, x_{v}, \xi, \eta\right]=-e^{3 \omega} \text {. }
$$

Since the Gauss maps $[\xi],[\eta]: M \rightarrow \mathbb{R} P^{3}$ are constant, we can find $\varphi, \psi \in$ $C^{\infty}(M)$ and constant vectors $\xi_{0}, \eta_{0} \in \mathbb{R}^{4}$ such that $\xi=e^{\varphi} \xi_{0}$ and $\eta=e^{\psi} \eta_{0}$. Since $\left\{x_{u}, x_{v}, \xi, \eta\right\}$ is a moving frame in $\mathbb{R}^{4}$ along $M$ and $x_{u v}$ is tangential, we can write the structure equations for $x$ in $\mathbb{R}^{4}$ as follows:

$$
\begin{array}{ll}
x_{u u}=\omega_{u} x_{u}+\xi, & x_{u v}=\lambda x_{u}+\mu x_{v} ; \\
x_{v u}=\lambda x_{u}+\mu x_{v}, & x_{v v}=\omega_{v} x_{v}+\eta ; \\
\xi_{u}=\varphi_{u} \xi, & \xi_{v}=\varphi_{v} \xi ; \\
\eta_{u}=\psi_{u} \eta, & \eta_{v}=\psi_{v} \eta ;
\end{array}
$$

where $\lambda, \mu \in \mathbb{C}^{\infty}(M)$. Using (3.5) and the identity $x_{u u v}=x_{u v u}$ we get

$$
\lambda=\varphi_{v}, \quad \omega_{u v}=\lambda_{u}+\lambda \mu, \quad \mu \omega_{u}=\mu_{u}+\mu^{2} .
$$

Using (3.6) and the identity $x_{v u v}=x_{v v u}$ we get

$$
\mu=\psi_{u}, \quad \omega_{u v}=\mu_{v}+\lambda \mu, \quad \lambda \omega_{v}=\lambda_{v}+\lambda^{2} .
$$

By (3.9) and (3.10) we get $(\varphi-\psi)_{u v}=0$. Thus we can find functions $f(u)$ and $g(v)$ on $M$ such that

$$
\varphi-\psi=f(u)-g(v)
$$

We note that the functions $\varphi$ and $\psi$ depend on the choices of asymptotic coordinate systems. If $\left(u^{*}, v^{*}\right)$ be another asymptotic coordinate system for $g$ with $u^{*}=u^{*}(u)$ and $v^{*}=v^{*}(v)$. Then we have the corresponding $\xi^{*}, \eta^{*}$ and functions $\varphi^{*}, \psi^{*}$ with $\xi^{*}=e^{\varphi^{*}} \xi_{0}, \eta^{*}=e^{\psi^{*}} \eta_{0}$. Sincne $\xi d u \otimes d u$ and $\eta d v \otimes d v$ are independent of the choices of asymptotic coordinate systems, we get $\varphi=\varphi^{*}+\ln \left(\frac{d u^{*}}{d u}\right)^{2}$ and $\psi=\psi^{*}+\ln \left(\frac{d v^{*}}{d v}\right)^{2}$. By (3.11) we have

$$
\varphi^{*}-\psi^{*}=f(u)-\ln \left(\frac{d u^{*}}{d u}\right)^{2}+\ln \left(\frac{d v^{*}}{d v}\right)^{2}-g(v)
$$

So we can always choose an asymptotic coordinate system $\left(u^{*}, v^{*}\right)$ such that $\varphi^{*}=\psi^{*}$. Such a system is uniquely determined up to constant factors.

Now we assume that $(u, v)$ is an asymptotic coordinate system with $\varphi=\psi$. By taking derivatives of (3.4) with respect to $u, v$ and using (3.9) and (3.10) we deduce

$$
3 \psi_{u}=2 \omega_{u}, \quad 3 \varphi_{v}=2 \omega_{v} .
$$

Thus we have $3 \varphi=3 \psi=2 \omega+2 c$ for some constant $c \in \mathbb{R}$. By taking the asymptotic coordinate systems $\left(e^{-c} u, e^{-c} v\right)$ if necessary we may assume that $c=0$. Thus we have

$$
3 \varphi=3 \psi=2 \omega
$$


From (3.9) and (3.10) we get

$$
\omega_{u v}=\frac{4}{3} \omega_{u} \omega_{v}, \quad \omega_{u u}=\frac{1}{3} \omega_{u}^{2}, \quad \omega_{v v}=\frac{1}{3} \omega_{v}^{2} .
$$

Using the identity $\omega_{u v u}=\omega_{u u v}$ we get $\omega_{u} \omega_{v}=0$. We claim that if $\omega_{u}(p)=0$ for some $p \in M$, then $\omega_{u} \equiv 0$ on $M$ and the same is true for $\omega_{v}$. In fact, we know from (3.15) that the function $t:=\omega_{u}$ satisfies the linear PDE system: $t_{v}=\frac{4}{3} \omega_{v} t, t_{u}=\frac{1}{3} \omega_{u} t$. Since $t \equiv 0$ is also a solution of it, if $\omega_{u}(p)=0=t(p)$, then $\omega_{u} \equiv 0$ on $M$. The same is clearly true for $\omega_{v}$. Thus $\omega_{u} \omega_{v}=0$ implies that (i) $\omega_{u}=\omega_{v} \equiv 0$ on $M$, or (ii) $\omega_{u} \equiv 0$ and $\omega_{v} \neq 0$, or (iii) $\omega_{u} \neq 0$ and $\omega_{v} \equiv 0$.

If (i) is true, i.e., $2 \omega=3 \varphi=3 \psi$ are constant, we know that the affine metric $g$ is flat. From (3.6) we get $x_{u v}=0$. Thus we can find 1 -variable vector-valued functions $\alpha$ and $\beta$ such that $x=\alpha(u)+\beta(v)$. By (3.5) and (3.6) we get

$$
\alpha^{\prime \prime}(u)=\xi, \quad \beta^{\prime \prime}(v)=\eta \text {. }
$$

Since $\xi$ and $\eta$ are constant vectors in $\mathbb{R}^{4}$, we obtain

$$
x=\frac{1}{2} u^{2} \xi+u \xi^{*}+\frac{1}{2} v^{2} \eta+v \eta^{*}+x_{0}
$$

for some constant vectors $\xi^{*}, \eta^{*}$ and $x_{0}$ in $\mathbb{R}^{4}$. Since $\left[\xi^{*}, \eta^{*}, \xi, \eta\right]=$ $\left[x_{u}, x_{v}, x_{u u}, x_{v v}\right] \neq 0$, we know that $x$ is affinely equivalent to the surface $\left\{\left(u, u^{2}, v, v^{2}\right) \mid(u, v) \in \mathbb{R}^{2}\right\}$ in $\mathbb{R}^{4}$.

Now we consider the cases (ii) and (iii). By exchanging $u$ and $v$ if necessary we may assume that (ii) is true, i.e., $\omega_{u} \equiv 0$ and $\omega_{v} \neq 0$ on $M$. By the last equation of (3.15) we know that $\omega$ is a function on $v$ such that $\omega_{v v}=\frac{1}{3} \omega_{v}^{2}$. Since $\omega_{v} \neq 0$, we get $\omega=-3 \ln (v-a)+3 b$ defined on $(a,+\infty)$ with arbitrary constants $a$ and $b$. From (3.5)-(3.8) and $\varphi=\psi=\frac{2}{3} \omega=-2 \ln (v-a)+2 b$ we get an integrable system

$$
\begin{gathered}
x_{u u}=(v-a)^{-2} e^{2 b} \xi_{0}, \quad x_{u v}=-2(v-a)^{-1} x_{u}, \\
x_{v v}=-3(v-a)^{-1} x_{v}+(v-a)^{-2} e^{2 b} \eta_{0} .
\end{gathered}
$$

From the first equation we get $x=\frac{1}{2}(v-a)^{-2} u^{2} e^{2 b} \xi_{0}+u \alpha(v)+\beta(v)$ for some 1-variable functions $\alpha$ and $\beta$. From the second equation of (3.17) we get $\alpha^{\prime}(v)=-2(v-a)^{-1} \alpha(v)$, which implies that $\alpha(v)=(v-a)^{-2} \xi_{1}$ for some constant vector $\xi_{1} \in \mathbb{R}^{4}$. From the last equation of (3.17) we get $\beta^{\prime \prime}(v)=-3(v-a)^{-1} \beta^{\prime}(v)+(v-a)^{-2} e^{2 b} \eta_{0}$ or equivalently $\left((v-a)^{3} \beta^{\prime}(v)\right)^{\prime}=$ $(v-a) e^{2 b} \eta_{0}$. Thus we get $\beta(v)=\frac{1}{2} \ln (v-a) e^{2 b} \eta_{0}+(v-a)^{-2} \eta_{1}+x_{0}$ for some constant vectors $\eta_{1}, x_{0} \in \mathbb{R}^{4}$. So we have

(3.18) $x=\frac{1}{2}(v-a)^{-2} u^{2} e^{2 b} \xi_{0}+(v-a)^{-2} u \xi_{1}+\frac{1}{2} \ln (v-a) e^{2 b} \eta_{0}+(v-a)^{-2} \eta_{1}+x_{0}$.

Since $\left[x_{u}, x_{v}, x_{u u}, x_{v v}\right]=2(v-a)^{-9} e^{4 b}\left[\xi_{1}, \eta_{0}, \xi_{0}, \eta_{1}\right] \neq 0$, we know that $x$ is affinely equivalent to the surface $\left\{\left(\ln (v-a),(v-a)^{-2},(v-a)^{-2} u\right.\right.$, $\left.\left.(v-a)^{-2} u^{2}\right) \mid(u, v) \in \mathbb{R}^{2}\right\}$ in $\mathbb{R}^{4}$. By taking $t=\ln (v-a)$ we get the surface (ii) in Theorem 2. Thus we complete the proof of Theorem 2.

\section{The PROOF OF THEOREM 3}

In this section, we will use the normalization discovered by Nomizu and the first author in [6]. We will closely follow the notation introduced there. Let $M$ be an indefinite surface in $\mathbb{R}^{4}$. We denote the Nomizu-Vrancken normal plane 
by $v$. It then follows from $\S 4$ of [6] that there exist local tangent vector fields $X_{1}, X_{2}$ and local normal vector fields $\xi_{1}, \xi_{2}$ such that

$$
\begin{gathered}
{\left[X_{1}, X_{2}, \xi_{1}, \xi_{2}\right]=1,} \\
h^{1}\left(X_{1}, X_{1}\right)=1, \quad h^{1}\left(X_{1}, X_{2}\right)=h^{1}\left(X_{2}, X_{2}\right)=0, \\
h^{2}\left(X_{2}, X_{2}\right)=1, \quad h^{2}\left(X_{1}, X_{2}\right)=h^{2}\left(X_{1}, X_{1}\right)=0,
\end{gathered}
$$

where $h^{1}$ and $h^{2}$ are the second fundamental forms of the immersion. From the definition of the affine metric $g$, it is clear that $\left\{X_{1}, X_{2}\right\}$ form a null-basis for $g$. Let $\left\{Y_{1}, Y_{2}, \tilde{\xi}_{1}, \tilde{\xi}_{2}\right\}$ be another basis which also satisfies (4.1); then if necessary by interchanging $Y_{1}$ and $Y_{2}$ or $\tilde{\xi}_{1}$ and $\tilde{\xi}_{2}$, we have

$$
Y_{1}=\lambda X_{1}, \quad Y_{2}=\lambda^{-1} X_{2}, \quad \tilde{\xi}_{1}=\lambda^{2} \xi_{1}, \quad \tilde{\xi}_{2}=\lambda^{-2} \xi_{2},
$$

where $\lambda$ is a positive local function. The above implies that the line bundles determined by $\xi_{1}$ and $\xi_{2}$ are well determined. These line bundles are called the affine Gauss maps with respect to the Nomizu-Vrancken normalization.

Let us now assume that these affine Gauss maps are constant. By (4.2), we then may assume that $\xi_{1}$ is constant and

$$
\xi_{2}=\varphi \eta
$$

where $\eta$ is a constant vector. But then

$$
D_{X} \xi_{2}=\varphi^{-1} d \varphi \xi_{2}
$$

However, since the Nomizu-Vrancken normalization is equiaffine, we have $\tau_{1}^{1}+$ $\tau_{2}^{2}=0$ and hence $\varphi$ is a constant. Thus $\xi_{2}$ is a constant vector as well. So we have shown the following lemma:

Lemma 4.1. Let $M$ be an indefinite surface with constant affine Gauss maps with respect to the Nomizu-Vrancken normalization. Then there exist a local basis $\left\{X_{1}, X_{2}\right\}$ and constant vector fields $\xi_{1}$ and $\xi_{2}$ such that

$$
\begin{array}{ll}
D_{X_{1}} X_{1}=\nabla_{X_{1}} X_{1}+\xi_{1}, & D_{X_{1}} X_{2}=\nabla_{X_{1}} X_{2}, \\
D_{X_{2}} X_{1}=\nabla_{X_{2}} X_{1}, & D_{X_{2}} X_{2}=\nabla_{X_{2}} X_{2}+\xi_{2}, \\
D_{X_{i}} \xi_{j}=0 . &
\end{array}
$$

A straightforward computation, using the fact that $\nabla$ is equiaffine and the Codazzi equation for $h$, we find that

$$
\begin{array}{lll}
\nabla_{X_{1}} X_{1}=\alpha_{1} X_{1}, & \nabla_{X_{1}} X_{2}=-2 \alpha_{2} X_{1}-\alpha_{1} X_{2}, \\
\nabla_{X_{2}} X_{1}=-\alpha_{2} X_{1}-2 \alpha_{1} X_{2}, & \nabla_{X_{2}} X_{2}=\alpha_{2} X_{2},
\end{array}
$$

where $\alpha_{1}$ and $\alpha_{2}$ are local functions. It now follows from the Gauss equation that there exists a function $f$ such that $\alpha_{1}$ and $\alpha_{2}$ satisfy the following system of differential equations:

$$
\begin{array}{ll}
X_{1}\left(\alpha_{1}\right)=3 \alpha_{1}^{2}, & X_{2}\left(\alpha_{1}\right)=3 \alpha_{1} \alpha_{2}+f, \\
X_{1}\left(\alpha_{2}\right)=3 \alpha_{1} \alpha_{2}-f, & X_{2}\left(\alpha_{2}\right)=3 \alpha_{2}^{2} .
\end{array}
$$

Lemma 4.2. The function $f$, defined above, is identically zero. 
Proof. We first look at the integrability condition for $\alpha_{1}$. This gives us

$$
\begin{aligned}
0= & X_{1}\left(X_{2}\left(\alpha_{1}\right)\right)-X_{1}\left(X_{2}\left(\alpha_{1}\right)\right)-\left(\nabla_{X_{1}} X_{2}-\nabla_{X_{2}} X_{1}\right)\left(\alpha_{1}\right) \\
= & 9 \alpha_{1}^{2} \alpha_{2}+3 \alpha_{1}\left(3 \alpha_{1} \alpha_{2}-f\right)+X_{1}(f)-6 \alpha_{1}\left(3 \alpha_{1} \alpha_{2}+f\right) \\
& +3 \alpha_{2} \alpha_{1}^{2}-\alpha_{1}\left(3 \alpha_{1} \alpha_{2}+f\right) \\
= & X_{1}(f)-10 \alpha_{1} f .
\end{aligned}
$$

Similarly, we find from the integrability condition for $\alpha_{2}$ that $X_{2}(f)=10 \alpha_{2} f$. The integrability condition for $f$ now yields

$$
\begin{aligned}
0= & X_{1}\left(X_{2}(f)\right)-X_{2}\left(X_{1}(f)\right)-\left(\nabla_{X_{1}} X_{2}-\nabla_{X_{2}} X_{1}\right)(f) \\
= & 10 f\left(3 \alpha_{1} \alpha_{2}-f\right)+100 \alpha_{2} \alpha_{1} f-100 \alpha_{1} \alpha_{2} f-10 f\left(3 \alpha_{1} \alpha_{2}+f\right) \\
& +10 \alpha_{1} \alpha_{2} f-10 \alpha_{1} \alpha_{2} f \\
= & -20 f^{2} .
\end{aligned}
$$

Hence $f$ vanishes identically. Q.E.D.

Now let us assume that there is a point $p$ such that $\alpha_{1}(p)=\alpha_{2}(p)=0$. Then it follows from (4.5) that $\alpha_{1}$ and $\alpha_{2}$ are zero in a neighbourhood of $p$. Hence it follows from (4.4) that we can choose local coordinates $(u, v)$ such that $X_{1}=\partial_{u}$ and $X_{2}=\partial_{v}$. Integrating then (4.3) gives us that $M$ is an open part of $\left(u, v, u^{2}, v^{2}\right)$.

Therefore we may assume that $\alpha_{2}(p) \neq 0$. Then we have

$$
X_{1}\left(\frac{\alpha_{1}}{\alpha_{2}}\right)=\frac{3 \alpha_{1}^{2} \alpha_{2}-3 \alpha_{1}^{2} \alpha_{2}}{\alpha_{2}^{2}}=0, \quad X_{2}\left(\frac{\alpha_{1}}{\alpha_{2}}\right)=\frac{3 \alpha_{2}^{2} \alpha_{1}-3 \alpha_{2}^{2} \alpha_{1}}{\alpha_{2}^{2}}=0 .
$$

So there exists a constant $c$ such that $\alpha_{1}=c \alpha_{2}$. It is then clear that, by taking $\lambda$ to be a constant in (4.2), we may assume that $c=0,1,-1$.

First we consider the case that $c=0$. Then the integrability conditions of

$$
X_{1}(\rho)=0, \quad X_{2}(\rho)=-\rho \alpha_{2}
$$

are trivially satisfied. Let $\rho$ be a solution of the above system. Then

$$
\left[\rho X_{1}, X_{2}\right]=-2 \alpha_{2} \rho X_{1}-X_{2}(\rho) X_{1}+\rho \alpha_{2} X_{1}=0 .
$$

So there exist coordinates $u$ and $v$ such that $x_{u}=\rho X_{1}, x_{v}=X_{2}$. It is then clear that $\rho$ and $\alpha_{2}$ only depend on the variable $v$ and are determined by

$$
\left(\alpha_{2}\right)_{v}=3 \alpha_{2}^{2}, \quad \rho_{v}=-\rho \alpha_{2} .
$$

Solving the first equation, after translating in the $v$ direction to eliminate the integration constant, we find that $\alpha_{2}=-\frac{1}{3 v}$. Hence $\rho(v)=v^{1 / 3}$ is a solution of the second equation. Integrating the system (4.3) then gives the surface given by $(1.10)$.

Next, we consider the case that $c=1$. In a similar manner as above, we find that there exist coordinates $u$ and $v$ such that

$$
x_{u}=X_{1}+X_{2}, \quad x_{v}=\rho\left(X_{1}-X_{2}\right),
$$

where $\alpha_{1}=-\frac{1}{6 u}$ and $\rho=u^{1 / 3}$. Integration of (4.3) then gives the surface given by $(1.9)$. 
Finally the case that $c=-1$ can be reduced to the previous one by replacing $X_{1} \rightarrow-X_{1}$ and by interchanging 2 coordinates in $\mathbb{R}^{4}$. This completes the proof of Theorem 3.

\section{ACKNOWLEDGMENTS}

We thank U. Simon for helpful discussions. Part of this work was done while the first author visited the TU Berlin in November 1992; he thanks the members of the department of mathematics for their hospitality.

\section{REFERENCES}

1. C. Burstin and W. Mayer, Die Geometrie zweifach ausgedehnter Mannigfaltigkeiten $F_{2}$ im affinen Raum $R_{4}$, Math. Z. 26 (1927), 373-407.

2. F. Dillen, G. Mys, L. Verstraelen, and L. Vrancken, The affine mean curvature vector for surfaces in $\mathbb{R}^{4}$, Math. Nachr. 166 (1994), 155-165.

3. W. Klingenberg, Zur affinen Differentialgeometrie, Teil I: Über p-dimensionale Mimimalflächen und Sphären im n-dimensionalen Raum, Math. Z. (1951), 65-80.

4. __ Zur affinen Differentialgeometrie, Teil II: Über 2-dimensionale Flächen im 4-dimensionalen Raum, Math. Z. 54 (1951), 184-216.

5. J. Li, Harmonic surfaces in affine 4-space, preprint.

6. K. Nomizu and L. Vrancken, A new equiaffine theory for surfaces in $\mathbb{R}^{4}$, Internat. J. Math. 4 (1993), 127-165.

7. C. Scharlach and L. Vrancken, Affine transversal planes for surfaces in $\mathbb{R}^{4}$, Geometry and Topology of Submanifolds V, World Scientific, Singapore, pp. 249-254.

8. R. Walter, Über zweidimensionale parabolische Flächen im vierdimensioalen affinen Raum, Teil I, Allgemeine Flächentheorie, J. Reine Angew. Math. 227 (1967), 178-208.

9. _ Über zweidimensionale parabolische Flächen im vierdimensioalen affinen Raum, Teil II, Spezille Flächen, J. Reine Angew. Math. 228 (1967), 71-92.

10. C. P. Wang, Surfaces in $\mathbb{R}^{4}$ with harmonic affine normal bundle, preprint.

(L. Vrancken) Departement Wiskunde, Katholieke Universiteit Leuven, CelestijnenLAAN 200 B, B 3001 LEUVEN, BELGIUM

E-mail address: Vrancken@davinci.wis.kuleuven.ac.be

(C. Wang) TU Berlin, FB-3, MA 8-3, Strabe des 17. Juni, 10623 Berlin, Germany

E-mail address: wang@math.tu-berlin.de

(C. Wang) Nankai Institute of Mathematics, Nankai University, Tianjing, 300071, PeoPLE'S REPUBLIC OF CHINA 